\title{
Investigation of 18F-FDG PET/CT Findings and CA-125 Levels in Ovarian Cancer Staging
}

\author{
(1) Reşit AKYEL, ${ }^{1}$ (D) Elife AKGÜN, ${ }^{2}$ (1) Burak AKOVALI, ${ }^{3}$ (D) Onur Erdem ŞAHIN, ${ }^{4}$ (I) Sait SAĞER, ${ }^{5}$ \\ (1) Abdullah Serdar AÇIKGÖZ, ${ }^{6}$ (1) Zübeyde Rana KAYA DÖNER, (1) Kerim SÖNMEZOĞLU 5
}

\author{
'Department of Nuclear Medicine, Yedikule Chest Diseases and Thoracic Surgery Training and Research Hospital, Istanbul-Turkey \\ 2Department of Nuclear Medicine, Kirikkale High Specialization Hospital, Kirikkale-Turkey \\ ${ }^{3}$ Department of Nuclear Medicine, Erzurum Regional Training and Research Hospital, Erzurum-Turkey \\ ${ }^{4}$ Department of Nuclear Medicine, Sivas Numune Hospital, Sivas-Turkey \\ ${ }^{5}$ Department of Nuclear Medicine, Istanbul University-Cerrahpasa, Cerrahpasa Faculty of Medicine, Istanbul-Turkey \\ ${ }^{6}$ Department of Gynecology and Obstetrics, Istanbul University-Cerrahpasa, Cerrahpasa Faculty of Medicine, Istanbul-Turkey \\ ${ }^{7}$ Department of Nuclear Medicine, Bursa Yuksek Ihtisas Training and Research Hospital, Bursa-Turkey
}

\begin{abstract}
OBJECTIVE
This study aims are to investigate the role of $18 \mathrm{~F}$ labeled fluoro-2-deoxy-D-glucose positron emission computed tomography/computed tomography (18F FDG PET/CT) in the initial staging and recurrence detection of ovarian cancer and to compare it with the cancer antigen-125 (CA-125) value.
\end{abstract}

\section{METHODS}

A total of 93 patients with a primary ovarian cancer diagnosis (Group $1 \mathrm{n}=41$ ) or suspicion of recurrent ovarian cancer (Group $2 \mathrm{n}=52$ ) were included in this study from January 2007 to January 2013.

\section{RESULTS}

The ages of the patients were between 15 and 82 years. In cases with PET positive lesions ( $\mathrm{n}=84$ ); Ovarian lesion $(n=34)$, infradiaphragmatic lymph node metastasis $(n=46)$, supradiaphragmatic lymph node metastasis $(n=14)$, peritoneal implant $(n=67)$, and distant metastasis $(n=14)$ were detected. Histopathological examination of 4 PET positive ovarian lesions was not compatible with cancer. In 14 cases with low CA-125 value, 18F FDG PET/CT was able to detect primary/recurrent lesions accurately. In the evaluation of primary/recurrent ovarian cancer, 18F FDG PET/CT had 93\% sensitivity, $42.8 \%$ specificity, $89.2 \%$ accuracy, whereas CA-125 had $79.1 \%$ sensitivity, $42.8 \%$ specificity, and $76.3 \%$ accuracy.

\section{CONCLUSION}

In conclusion; even in low CA-125 values, 18F FDG PET/CT is a prominent method that can detect especially extra-abdominal distant metastatic foci in the initial staging of primary ovarian cancer and diagnosis and follow-up of recurrent ovarian cancer.

Keywords: $18 \mathrm{~F}$ labeled fluoro-2-deoxy-D-glucose; cancer antigen-125; ovarian cancer; positron emission tomography/computed tomography; $\mathrm{SUV}_{\max }$.

Copyright $\odot$ 2022, Turkish Society for Radiation Oncology

\section{Introduction}

Ovarian cancer is the second most common gynecological malignancy after endometrial cancer, with an inci- dence of $25 \%$. About 1 in 100 women will develop the disease and two-thirds will die from their malignancy. It is more common in the post-menopausal period and $80 \%$ of the patients are over the age of 50 years.[1] 
The most important prognostic factor is the disease stage at the time of diagnosis. Although the grade and histology of the tumor are not important in general, small cell and clear cell subtypes affect the prognosis negatively.

Due to their rapid growth and early asymptomatic nature; at the time of diagnosis, $15 \%$ of the cases were at the local stages, $18 \%$ were at the regional stages, and $61 \%$ were at the advanced stages.[2] It is a gynecological cancer with the highest mortality rate.[3]

When all stages are evaluated, the 5-year survival rate is $44.2 \%$; $93 \%$ for Stage I, $70 \%$ for Stage II, 37\% for Stage III, and $25 \%$ for Stage IV.[4] In recurrent disease, the mortality rate is up to $80 \%$.

Although $80 \%$ of patients respond to surgery and adjuvant chemotherapy, recurrence is detected in $80 \%$ of patients at an advanced stage. Small residual tumor volume (1-2 $\left.\mathrm{cm}^{3}\right)$ after primary cytoreductive surgery is an important prognostic parameter in ovarian cancer surgery.[5]

$18 \mathrm{~F}$ labeled fluoro-2-deoxy-D-glucose positron emission computed tomography (CT)/CT (18F-FDGPositron Emission Tomography [PET]/CT), is a functional imaging method that provides information about both anatomy and metabolism and is used to determine restaging and treatment response in ovarian cancer. Our study aims are to investigate the importance of 18F-FDG PET/CT in the initial staging and in detecting the recurrence of ovarian cancer and compare it with Cancer antigen-125 (CA-125) levels.

\section{Materials and Methods}

\section{Study Population}

A total of 93 patients, with full clinical follow-up, who underwent F18-FDG PET/CT scans for ovarian cancer research between 2007 and 2013 were included in this study. The patients were divided into two groups as primary staging (Group 1, n=41) and recurrence (Group 2, $\mathrm{n}=52$ ).

Patients who had 18F-FDG PET/CT scan at short intervals for control purpose and had no clinical, laboratory, and radiological suspicion of the disease or received chemotherapy 3 weeks before the PET scan and radiotherapy within 3 months before PET scan have not been included in this study.

The patients' age, primary ovarian cancer subtype, grade, stage, CA- 125 value in the last month, and maximum standard uptake values $\left(\mathrm{SUV}_{\max }\right)$ of lesions accepted as PET-positive, were noted.

\section{F-FDG PET/CT Imaging}

PET/CT imaging of all cases was performed with a high-resolution PET scanner integrated with 6-slice CT (Siemens Biograph LSO HI-REZ PET/CT Illinois, USA). 444-629 MBq (12-17 mCi) F-18 FDG was injected intravenously, with a minimum of $4 \mathrm{~h}$ of fasting and blood glucose level below $160 \mathrm{mg} / \mathrm{dl}$. After FDG injection, the patients were rested in a quiet room for 1-1.5 $\mathrm{h}$ to complete the biodistribution of the radiopharmaceutical. After the bladder emptied, the patients were placed in the supine position on the PET/CT scanner. After the topogram, non-contrast low-dose CT and PET images $(3 \mathrm{~min} / \mathrm{bed})$ from the vertex to the mid of the thigh were obtained. PET images were reconstructed with iterative configuration and CT images were used for attenuation correction.

\section{Interpretation of 18F-FDG PET/CT Data}

PET, CT, and PET/CT fusion images were analyzed simultaneously. In visual evaluation, the main criterion for accepting it as a lesion was the detection of increased focal FDG uptake compared to background activity. Lesion equivalent of each focal FDG uptake was investigated on corresponding CT images. Focal FDG involvements corresponding to salivary gland, muscle, adipose tissue, and reactive lymph node on CT scans were considered as physiological involvements. Any focal FDG uptake detected in ascites, abnormal soft tissue mass, or pathological lymph node was considered as a lesion. Mild FDG involvement with signs of infection/inflammation on CT images was not considered as recurrence/metastasis. Semi-quantitative $\mathrm{SUV}_{\max }$ was calculated from the Region of Interest drawn over the focus considered as a lesion in both PET and CT.

\section{Statistical Analysis}

Number Cruncher Statistical System 2007 and Power Analysis and Sample Size 2008 Statistical Software (Utah, USA) program was used for statistical analysis. Student's t-test and Mann Whitney U test were used according to the distribution pattern of the data. While Fisher's exact test and Yates Continuity Correction test were used to compare qualitative data, ROC curve analysis was used to determine the cutoff value. Statistical significance was accepted as $\mathrm{p}<0.05$.

\section{Results}

The mean age at the time of diagnosis was 54.4 years (max: 83, min: 15). 45\% $(n=42)$ of the cases were in the premenopausal period, and $55 \%(\mathrm{n}=51)$ were in 
the postmenopausal period. The time between the last treatment and PET scan of patients being investigated for recurrence or metastasis; ranged from 3 weeks to 70 months. Group 2 developed recurrence after therapies (chemotherapy/cytoreductive surgery/both) at an average of 16 months.

The verification of the lesions considered as suspicious in PET/CT was performed histopathologically in 28 patients in Group 1 and 17 patients in Group 2 (48.4\% of all cases). In the remaining, verification was performed with clinical follow-up, CA-125 levels, conventional imaging methods, and control PET/CT images.

According to the clinical follow-up, CA-125 values, control imaging, and histopathologic examination; primary/recurrent ovarian cancer was detected in 86 patients $(92.5 \%)$, benign lesions were detected in 4 patients (4.3\%). Three patients $(3.2 \%)$ had no malignant lesions (Table 1).
Table 1 Histopathological diagnosis of cases with malignancy

\begin{tabular}{lcc} 
Histopathological diagnosis & $\mathbf{n}$ & \% \\
\hline Serous papillary carcinoma & 61 & 71 \\
Mix type epithelial carcinoma & 8 & 9.3 \\
Clear cell carcinoma & 2 & 2.3 \\
Germ cell carcinoma & 7 & 8.1 \\
Musinous carcinoma & 3 & 3.5 \\
Malign mix mullerian tumor & 2 & 2.3 \\
Low differentiated carcinoma & 2 & 2.3 \\
Endometrioid type adenocarcinoma & 1 & 1.2 \\
\hline
\end{tabular}

When four false-positive cases in Group 1 were evaluated histopathologically, tuberculosis in two patients, amyloidosis in one patient, and serous cystadenoma in one patient were found (Fig. 1).

According to PET images, total of six false-negative cases were detected. PET could not detect lesions in $2 \%$

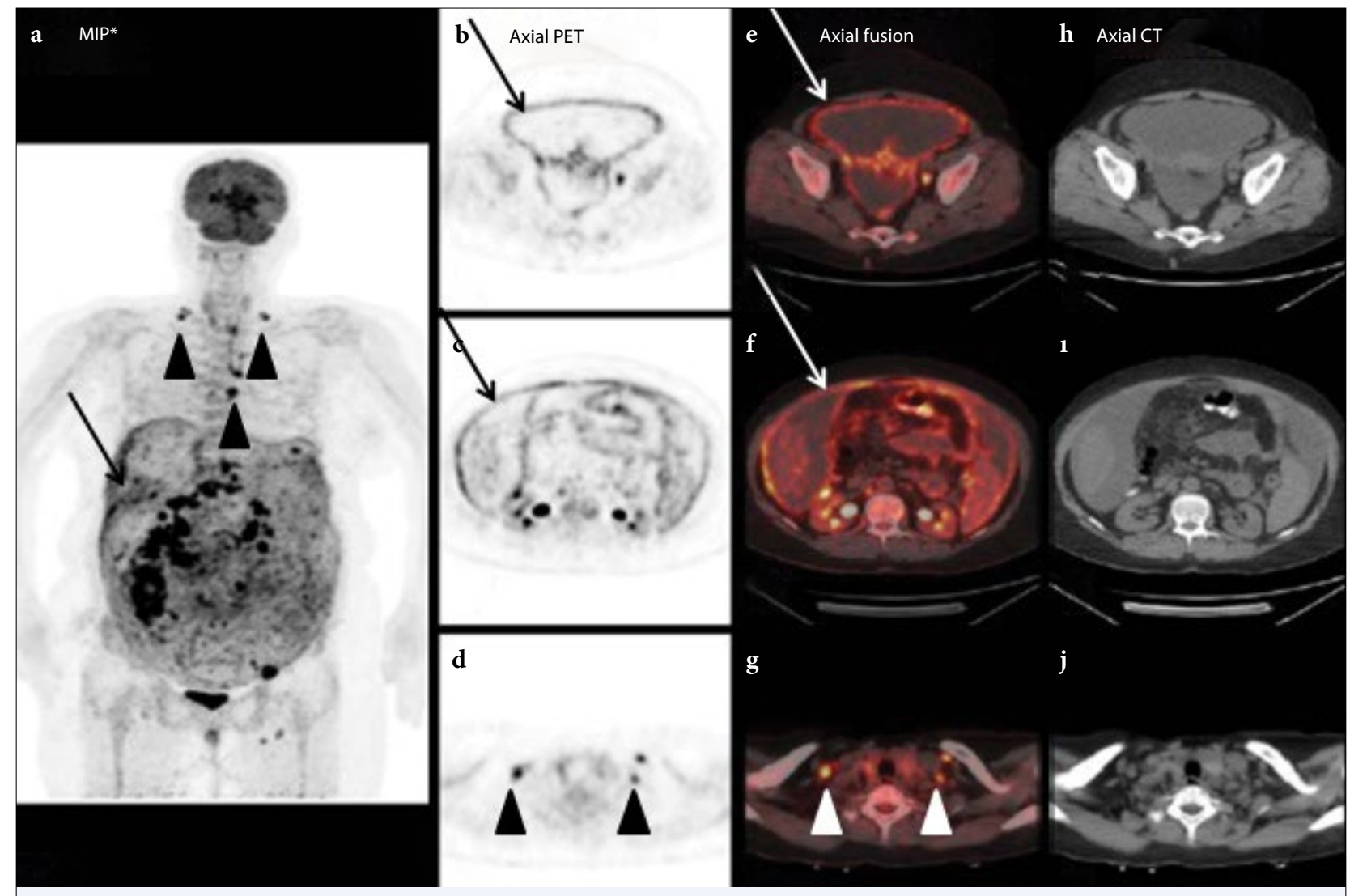

Fig. 1. Maximum intensity projection (MIP) image of Positron emission tomography (PET) images of the patient (arrow and arrowheads in a), who underwent scan for high CA-125 level shows the disease spread. Axial images of PET (arrows in b and c) and axial fused PET/CT (Positron emission tomography/Computed tomography) (arrows in e and f) show peritoneal spread of disease. Bilateral supraclavicular hypermetabolic lymph nodes in PET (arrowheads in $\mathrm{d}$ ) and PET/CT fusion images (arrowheads in $\mathrm{g}$ ). The locations of FDG avid tissues can be clearly determined anatomically via CT images $(\mathrm{h}-\mathrm{j})$.

${ }^{\star}$ Maximum intensitiy projection (MIP). Positron emission tomography/Computed tomography (PET/CT); CA: Cancer antigen. 
of postmenopausal patients and $4 \%$ of premenopausal patients as false-negative. Two of these six patients had a history of neoadjuvant chemotherapy. In four of them, it has been noted that the lesions did not cause a mass effect in the ovary and there was quite a low FDG uptake in normal-sized ovaries.

The accuracy rate reached $92 \%$ in Group 1. When all cases were evaluated, the sensitivity, specificity, positive predictive value (PPV), negative predictive value (NPV), and accuracy rates of FDG-PET/CT were $93 \%$, $42.8 \%, 95.2 \%, 33.3 \%$, and $89.2 \%$, respectively.

Lesion localizations on FDG-PET/CT images of 84 patients were as follows: primary ovarian cancer in 37 patients, infradiaphragmatic lymph node metastasis in 46 patients, supradiaphragmatic lymph node metastasis in 14 patients, peritoneal implant in 67 patients, and distant organ metastasis in 14 patients. Implants were located mostly in ovarian neighborhoods, large intestine serosa, and liver-spleen capsular surfaces. PET was able to detect the lesion in $82.5 \%$ of patients with primary malignancy. The presence of nodal disease in primary and recurrent lesions was $65 \%$ and $70 \%$, respectively. Serous and endometrioid histology were found to be independent predictors of nodal metastases, but other advanced ovarian cancer patients who did not belong to these pathologies also had nodal metastases.

In Group 1, the mean ovarian lesion $\mathrm{SUV}_{\text {max }}$ is 15.4 (serous papillary CA SUV max $_{1}: 15.5$, endometrioid CA $\mathrm{SUV}_{\max }: 12$, clear cell CA SUV $\mathrm{max}_{\max }: 4.5$, mucinous ovary $\mathrm{CA} \mathrm{SUV}_{\max }: 5.8$, Germ cell CA SUV $\left.\mathrm{max}_{\max }: 2.4\right)$. The mean $\mathrm{SUV}_{\max }$ was 13.8 for peritoneal implants, 13.9 for lymph node metastases, and 2.3 for peritoneal acid. As the $\mathrm{SUV}_{\max }$ value of the primary lesion increased, the SUV${ }_{\max }$ value of the implant also increased $(\mathrm{r}=0.64$, Fig. 2$)$.

CA- 125 value was below normal in 21 cases $(<35 \mathrm{U} /$ $\mathrm{ml}$ ) and above normal in 72 cases (mean: $1460 \mathrm{U} / \mathrm{ml}$, max: $18000 \mathrm{U} / \mathrm{ml}$ ). Of the 21 patients with low CA-125 levels, 18 of them had proven malignancy. Four of 72 cases with elevated CA- 125 value were negative for malignancy with radiologic imaging and clinical followup. The sensitivity, specificity, PPV, NPV, and accuracy rates of CA- 125 were $79.1 \%, 42.8 \%, 94.4 \%, 14.3 \%$, and $76.3 \%$, respectively. PET/CT could not detect lesions in $6 \%$ of patients with elevated CA- 125 .

\section{Discussion}

Despite clinical developments and advanced surgical techniques, ovarian cancer is the most mortal gynecological cancer, due to its nonspecific symptoms and advanced-stage diagnosis. Surgery is considered in the

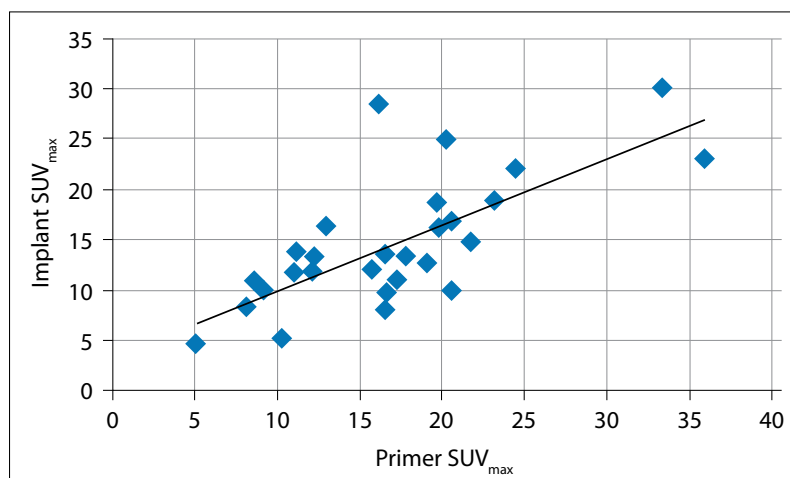

Fig. 2. Primary lesion and implant's $S U V_{\text {max }}$ value relationship. SUV ${ }_{\max }$, Maximum standart uptake value. SUV: Standard uptake values.

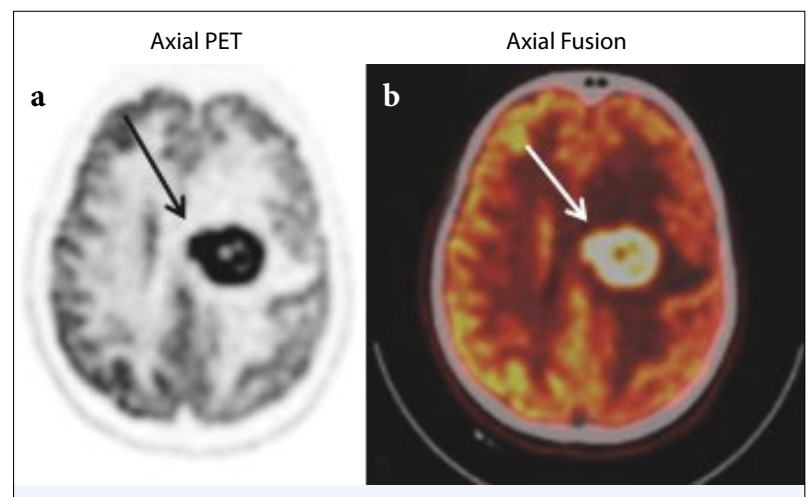

Fig. 3. Brain metastasis was detected in an axial PET image of the patient who was screened for the initial staging of ovarian cancer (arrow in a). Axial fused Positron emission tomography/computed tomography (PET/CT) image of the patient shows the metastasis more clearly (arrow in b). According to the brain Magnetic resonance imaging findings and brain biopsy histopathologic examination, this lesion was confirmed as ovarian cancer metastasis. PET: Positron emission tomography.

first-line therapy since tumor burden is an important parameter in treatment, and then the disease is tried to be controlled with systemic chemotherapy.[3]

Although ovarian cancer predominantly metastases to the peritoneal cavity and pelvic lymph nodes, recent evidence suggests the possibility of hematogenous metastasis of ovarian cancer. Implants are mostly localized in the pelvis, right hemidiaphragm, liver capsular surface, right paracolic area, intestines, and omentum. [6] It has been reported in the literature that $10-25 \%$ of patients have pelvic recurrences, and the incidence of recurrence in lymph nodes is $26 \%$.[7] In our study, $75 \%(n=39)$ of patients in Group 2 had peritoneal implants, 52\% $(n=27)$ of patients had pelvic-paraaortic lymph nodes metas- 


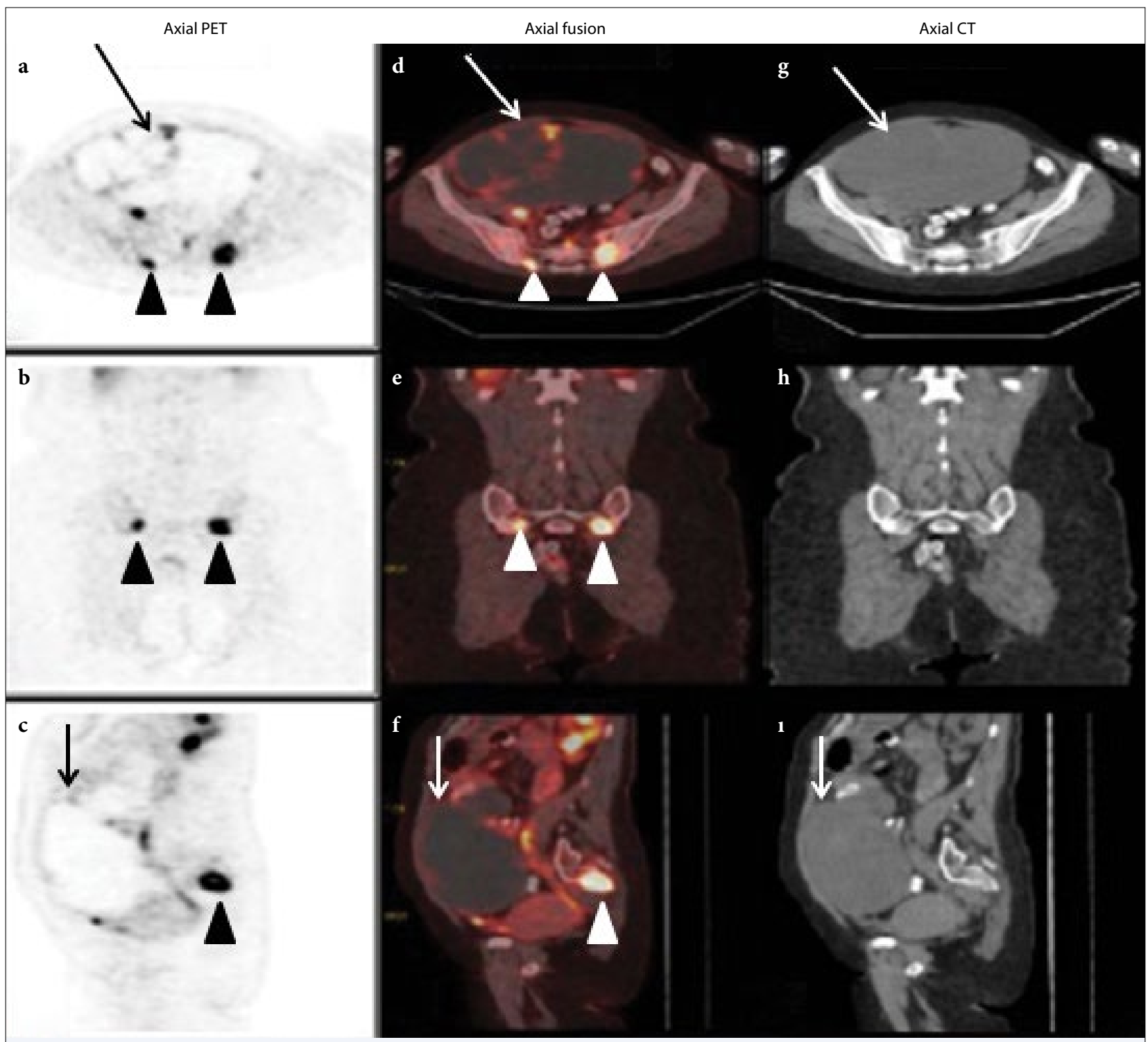

Fig. 4. Axial PET (arrow in a), sagittal PET (arrow in c) and Positron emission computed tomography/Computed tomography fused images (arrows in $\mathrm{d}$ and $\mathrm{f}$ ) of the patient, who was investigated for ovarian malignancy due to high CA-125 level $(230 \mathrm{U} / \mathrm{ml})$ and pelvic mass, revealed a peripheral hypermetabolic cystic lesion that filled the pelvis, compatible with primary ovarian malignancy. Hypermetabolic lesions compatible with sacral bone metastases (arrowheads in $\mathrm{b}$ and $\mathrm{e}$ ). These lesions were confirmed as primary ovarian cancer and its bone metastasis histopathologically. CT images ( $\mathrm{g}, \mathrm{h}$ and $\mathrm{i}$ ) help in anatomical localization.

PET: Positron emission tomography; CT: Computed tomography; CA: Cancer antigen.

tasis, $11.5 \%(\mathrm{n}=6)$ of patients had supradiaphragmatic lymph node metastases, $9 \%(n=6)$ of patients had distant metastasis (two brain, one lung, one liver, and one bone metastasis) (Figs. 3-5). The difference with the literature can be explained by the fact that our results are only for the patient group investigated for recurrence.

FDG-PET/CT is superior to other imaging methods in detecting distant organ or lymph node metastasis in primary ovarian cancer. It has shown that patients with supradiaphragmatic lymph node metastases develop more frequent ascites. That confirms the information that tumor cells cross the diaphragm mainly by cardiophrenic lymph nodes and parasternal lymphatics. [8] In our series, the supradiaphragmatic lymph node metastasis rate was found to be $11.5 \%$ in the group whose recurrence was investigated.

Due to the high incidence of recurrence in ovarian cancers, clinical follow-up is very important; there- 


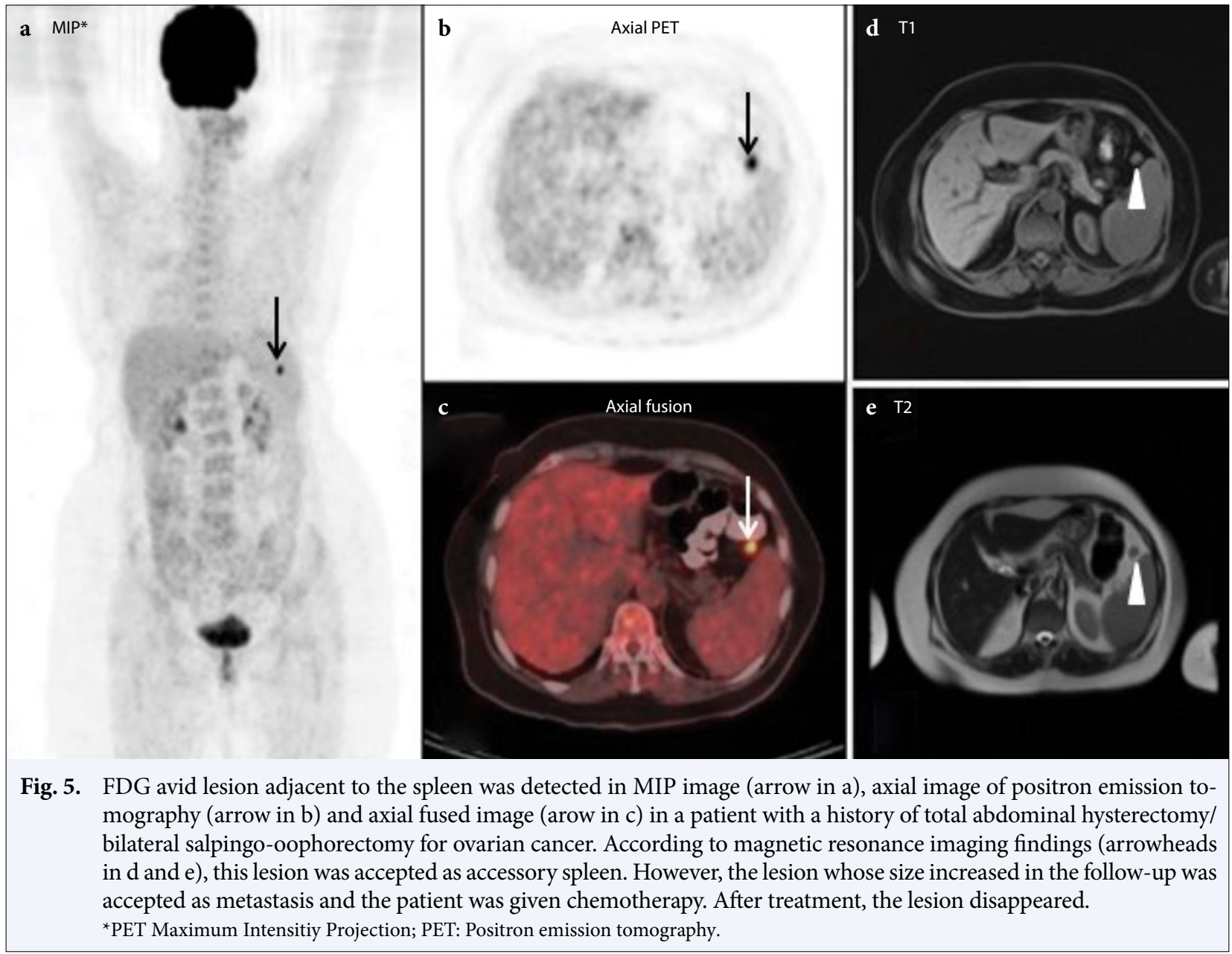

fore, CA-125 is a very useful parameter. Von Georgi et al.,[7] detected high CA-125 levels in $80 \%$ of ovarian cancer patients. In the study of Chang et al.,[9] although recurrence was detected in $95-100 \%$ of cases with high CA-125 level, low CA-125 level was found in approximately $50 \%$ of the cases with recurrence. The literature revealed that $46 \%$ of patients with normal CA-125 values had the disease in second-look surgery.[10] While the specificity of CA-125 value in our study was calculated as $42.8 \%$, similar to the literature, the sensitivity was slightly lower than the literature with $79.1 \%$. In our study, high CA-125 levels were found in 68 (79\%) patients whose primary/ recurrent disease was clinically or histopathologically proven. However, the CA-125 value of 18 patients was lower than $35 \mathrm{U} / \mathrm{ml}$. In addition, CA-125 values were found to be high in 4 cases with FDG positive ovarian lesions and histopathological findings were found to be consistent with cystadenoma, tuberculosis, and amyloidosis. This finding suggests that CA-125 is not a cancer-specific marker.

$\mathrm{PET} / \mathrm{CT}$ can detect early recurrences even with low CA-125 value ( $<35 \mathrm{U} / \mathrm{ml})$. Palomar et al.,[11] showed that decreasing the CA-125 cut-off value to $18 \mathrm{U} / \mathrm{ml}$ achieves a high recurrence detection rate. In our study, although CA- 125 values were low, FDG-PET/CT clearly showed the location of the lesions in 14 patients with primary / recurrent tumor. The sensitivity, specificity, PPV, NPV, and accuracy of FDG PET/CT for detection of ovarian cancer was $93 \%, 42.8 \%, 95.2 \%, 33.3 \%$, and $89.2 \%$, respectively; compared to $79.1 \%, 42.8 \%, 94.4 \%$, $14.3 \%$, and $76.9 \%$ for CA-125. Therefore, in necessary cases, PET/CT imaging can be used even with low tumor markers.

CT is the first imaging method used in the follow-up of patients and evaluation of relapse. In the literature, the sensitivity of CT in detecting relapses has been reported as $40-93 \% .[12,13]$ In patients with a complete clinical 
response to primary therapy and an increase in CA-125 levels in their follow-up, lesions may not be detected by CT. Morphological features of lesions such as shape, size, and contrast enhancement are important in detecting recurrent disease in CT. CT alone may not be sufficient to detect local recurrence, pelvic lymph node metastasis (especially those located very close to the intestinal structures), or postoperative scar tissue.[14] The most common form of recurrent disease is peritoneal spread.

Diagnosis of peritoneal implants by radiological methods has been a problem due to their small size and location. In the study of De Rosa et al.,[15] comparing the results of CT with second-look laparotomy, the sensitivity and specificity of CT were found to be $47 \%$ and $87 \%$, respectively. In another study by Makhija et al.,[16] while PET/CT was positive in 5 of 8 patients (62\%) with histopathologically proven recurrent disease, CT was negative. When we compared conventional imaging methods with PET/CT images, while CT could not detect recurrent foci in 11 of 15 patients (73\%), FDG-PET/CT showed all these recurrences. FDG-PET/CT is a whole-body functional imaging that can provide valuable information in staging and diagnosis of ovarian cancers. It was also revealed that FDGPET/CT detects more lesions than CT.[17,18] Accurate and complete pre-operative detection of lesions in patients scheduled for surgical treatment, contributes greatly to patient management.

In the study by Chung et al.,[19] involving 77 patients with suspected recurrent ovarian cancer, the sensitivity of PET/CT was $93.3 \%$, specificity was $96.9 \%$ and accuracy was $94.8 \%$, respectively. The reason for the low specificity is attributed to the prevalence of diseases with high FDG affinity in our country, such as amyloidosis and sarcoidosis.

It has been shown that FDG PET/CT can give false-negative results in the early period of the disease and in the detection of recurrences in mucinous subtypes of ovarian cancer.[20] In the study of Yuko et al.,[21] increased FDG accumulation was observed in 6 of $11(54.5 \%)$ clear cell subtype ovarian cancers and in 4 of $6(66.7 \%)$ mucinous subtype ovarian cancers. In the same study, the mean $\mathrm{SUV}_{\max }$ value was found to be 6.9 in the serous subtype, 3.5 in the clear cell subtype, and 3.4 in the mucinous cell subtype. SUV${ }_{\text {max }}$ values of serous ovarian cancer were found to be significantly higher than clear cell $(\mathrm{p}<0.01)$ and mucinous cell subtypes $(\mathrm{p}<0.03)$. In our series, the mean $\mathrm{SUV}_{\max }$ value of the primary ovarian lesion was 15.4 and the mean value of serous and endometrioid types were significantly higher than other histological sub- types $(\mathrm{p}<0.05)$. The lowest FDG avidity was found in the germ cell type.

Our study is valuable in terms of including a large number of patients and only cases with suspected disease.

\section{Conclusion}

FDG PET is a non-invasive imaging method with high sensitivity and accuracy in the detection and staging of primary/recurrent ovarian cancer, useful in post-treatment follow-up, and allowing metabolic and anatomical imaging in a single session.

Although peritoneal implants and metastatic paraaortic lymph nodes were detected in most cases in our study, mediastinal, supraclavicular, and cervical metastatic lymph nodes and distant organ metastases were also detected in some cases and very important information was given to the clinicians about the current status of the disease.

FDG-PET/CT, which can detect extra-abdominal distant metastatic lesions, in particular, was found to be more sensitive and important in the follow-up and treatment of the disease than CA-125.

Acknowledgments: All authors have read and agreed with the content of the manuscript. Each author has participated sufficiently in the work to take public responsibility for appropriate portions of the content.

Peer-review: Externally peer-reviewed.

Conflict of Interest: All authors declared no conflict of interest.

Ethics Committee Approval: The study was approved by The İstanbul University-Cerrahpaşa, Cerrahpaşa Faculty of Medicine Ethics Committee (No: 83045809/604/02-8850, Date: $04 / 04 / 2014$ ).

Financial Support: This study has received no financial support.

Authorship contributions: Concept - R.A., E.A., ; Design - E.A.; Supervision - S.S., K.S.; Funding - R.A., E.A.; Materials - R.A., O.E.Ş.; Data collection and/or processing - E.A., B.A., Z.R.K.D.; Data analysis and/or interpretation - E.A.; Literature search - E.A., R.A.; Writing - E.A., R.A.; Critical review - K.S., A.S.A., S.S.

\section{References}

1. Rooth C. Ovarian cancer: risk factors, treatment and management. Br J Nurs 2013;22(17):23-30.

2. Siegel R, Ma J, Zou Z, Jemal A. Cancer statistics, 2014. CA Cancer J Clin 2014;64(1):9-29. 
3. Marcus C, Maxwell GL, Darcy KM, Hamilton CA, McGuire WP. Current approaches and challenges in managing and monitoring treatment response in ovarian cancer. J Cancer 2014;5(1):25-30.

4. Cass I, Baldwin RL, Varkey T, Moslehi R, Narod SA, Karlan BY. Improved survival in women with BRCA-associated ovarian carcinoma. Cancer 2003;97(9):2187-95.

5. Le T, Krepart GV, Lotocki RJ, Heywood MS. Does debulking surgery improve survival in biologically aggressive ovarian carcinoma. Gynecol Oncol 1997;67(2):208-14.

6. Oliphant M, Berne AS, Meyers MA. Bidirectional spread of disease via the subperitoneal space: the lower abdomen and left pelvis. Abdom Imaging. 1993;18(2):117-25.

7. von Georgi R, Schubert K, Grant P, Münstedt K. Posttherapy surveillance and after-care in ovarian cancer. Eur J Obstet Gynecol Reprod Biol 2004;114(2):228-33.

8. Johanna H, Auranen A, Carpén O, Dean K, Seppänen $M$, Kemppainen J, et al. FDG PET/CT in staging of advanced epithelial ovarian cancer: Frequency of supradiaphragmatic lymph node metastasis challenges the traditional pattern of disease spread. Gynecol Oncol 2012;126(1):64-8.

9. Chang WC, Hung YC, Kao CH, Yen RF, Shen YY, Lin CC. Usefulness of whole body positron emission tomography (PET) with 18F-fluoro-2-deoxyglucose (FDG) to detect recurrent ovarian cancer based on asymptomatically elevated serum levels of tumor marker. Neoplasma 2002;49(5):329-33.

10. Berek JS. Ovarian cancer. Novaks Gynecology. $13^{\text {th }}$ ed. Philadelphia: Lippincott Williams \& Wilkins; 2002. p. 1245-318.

11. Palomar A, Nani C, Castellucci P, Ambrosini V, Montini CG, Allegri V, et al. Value of FDG PET/CT in patients with treated ovarian cancer and raised CA125 serum levels. Mol Imaging Biol 2012;14(1):123-9.

12. Prayer L, Kainz C, Kramer J, Stiglbauer R, Schurawitzki $\mathrm{H}$, Baldt M, et al. CT and MR accuracy in the dedection of tumor recurrence in pations treated for ovarian cancer. J Comput Asist Tomogr 1993;17(4):626-32.

13. Bristow RE, Marcela GC, Pannu HK, Cohade C, Zahurak ML, Fishman EK, et al. Clinically occult recurrent ovarian cancer: patient selection for secondary cytoreductive surgery using combined PET/CT. Gynecol Oncol 2003;90(3):519-28.

14. Hauth EA, Antoch G, Stattaus J, Kuehl H, Veit P, Bockisch A, et al. Evaluation of integrated whole-body $\mathrm{PET} / \mathrm{CT}$ in the detection of recurrent ovarian cancer. Eur J of Radiol 2005;56(2):263-8.

15. DeRosa V, Mangoni di SML, Brunetti A, Caraco C, Graziano R, Gallo MS, et al. Computed tomography and secondlook surgery in ovarian cancer patients. Correlation, actual role and limitations of CT scan. Eur J Gynaecol Oncol 1995;16(2):123-9.

16. Makhija S, Howden N, Edwards R, Kelley J, Townsend DW, Meltzer CC. Positron emission tomography/ computed tomography imaging for the detection of recurrent ovarian and fallopian tube carcinoma: a retrospective review. Gynecol Oncol 2002;85(1):53-8.

17. Musto A, Rampin L, Nanni C, Marzola MC, Fanti S, Rubello D. Present and future of PET and PET/ $\mathrm{CT}$ in gynaecologic malignancies. Eur J Radiol 2011;78(1):12-20.

18. Thrall MM, DeLoia JA, Gallion H, Avril N. Clinical use of combined positron emission tomography and computed tomography (FDG-PET/CT) in recurrent ovarian cancer 2007;105(1):17-22.

19. Chung HH, Kang WJ, Kim WJ, Park NH, Song YS, Chung JK, et al. Role of [18F] FDG PET/CT in the assessment of suspected recurrent ovarian cancer: correlation with clinical or histological findings. Eur J Nucl Med Mol Imaging 2007;34(4):480-6.

20. Son H, Khan SM, Rahaman J, Cameron KL, PrasadHayes M, Chuang L, et al. Role of FDG PET/CT in staging of recurrent ovarian cancer. Radio Graphics 2011;31(2):569-83.

21. Tanizaki Y, Kobayashi A, Shiro M, Ota N, Takano R, Mabuchi $\mathrm{Y}$, et al. Diagnostic value of preoperative SUVmax on FDG-PET/CT for the detection of ovarian cancer. Int J Gynecol Cancer 2014;24(3):454-60. 